\title{
Desktop Virtual Maintenance Training Platform Based on NGRAIN
}

\author{
Wang Xu-ping ${ }^{1, a}$, Chen Xiao-hu' ${ }^{1, b}$, Yao Chun-jiang ${ }^{1, c}$, \\ Ding $\mathrm{Yao}^{1, \mathrm{~d}}$, Gao Shu-xiang ${ }^{1, \mathrm{e}}$ \\ ${ }^{1}$ Xi'an Research Inst. Of Hi-Tech Hongqing Town, Xi'an, Shanxi Province, China \\ axiaobing_king@163.com, ${ }^{\mathrm{b}}$ TigerChen@163.com, ${ }^{\mathrm{c}}$ yaochunjiang@163.com, \\ ddingyao.88@163.com, e gaoshuxiang88@126.com
}

Keywords: virtual maintenance training; NGRAIN; 3D interactive; COM control

\begin{abstract}
According to the current status of maintenance training, a framework of desktop virtual maintenance training platform was proposed on the basis of the NGRAIN ,making full use of advanced CAD technology and virtual reality technology. At last take the dump trucks as example, which proved that equipment virtual maintenance training can largely improve the cost-effectiveness of the maintenance training. The platform is a portable, economical solution for maintenance training, which not only can meet the needs of the training mission, but also provide a reference for the other virtual maintenance training system. In the future, the platform will have a very important practical significance and broad application prospects.
\end{abstract}

\section{Introduction}

Currently, troops and equipment maintenance training facing a serials problems, such as a single means of training, the training effect is not ideal and expensive maintenance training, which largely restricts the improvement of the combat effectiveness of troops. Therefore, a new training means is urgent needed to solve the current problems[1]. Virtual maintenance is based on the current actual of equipment maintenance and training, some attempts have been took for the actual application of virtual reality technology for maintenance training at domestic and abroad, and some good results have been achieved[2-6].

NGRAIN Company of Canada, a 3D interactive training solutions provider, which provides lightweight program for model rendering based on voxel, a accelerator card is not required when rendering graphics. The system can run on ordinary PC, portable machines and PDA. Therefore, a desktop virtual maintenance training platform based on NGRAIN can alleviate or even solve the problems that have occurred in the current maintenance training.

\section{Desktop virtual maintenance training platform technology framework}

Virtual Maintenance Training TQCSE characteristics[7]:Time, advance training opportunity, shorten the training time; Quality, function improved, significant training effect; Costs, effectively reducing the training costs; Safety, the safety of personnel and equipment; Environment of the , immune from the venue and time restrictions environmental conditions.

Virtual maintenance training is usually based on computer systems, integrated 3D modeling and computer simulation and virtual reality technology were implicated by equipment, repair tools, personnel, human-computer interaction process. According to using simulation to show maintenance information in a virtual environment, a maintenance environment is provided, participation-based and supplemented with a certain degree of autonomy, for trainees by Virtual Maintenance Training .It is good means to form, maintain, or improve the knowledge and skills of trained personnel and assessed their level.

NGRAIN itself is a desktop-level interactive training system development software, therefore ,ordinary users can quickly create and publish interactive virtual training courseware without programming or writing scripts, and then the training content can been configured in the HTML, DOC, PPT and PDF documents through the controls included in NGRAIN Viewer. The 
functional modules of the system are composed of six parts, including virtual prototype 3D modeling, model conversion module, 3D interactive content creation module, 3D interactive content to show module, ordinary show module, maintenance of the knowledge base. The specific framework shown in Fig.1.

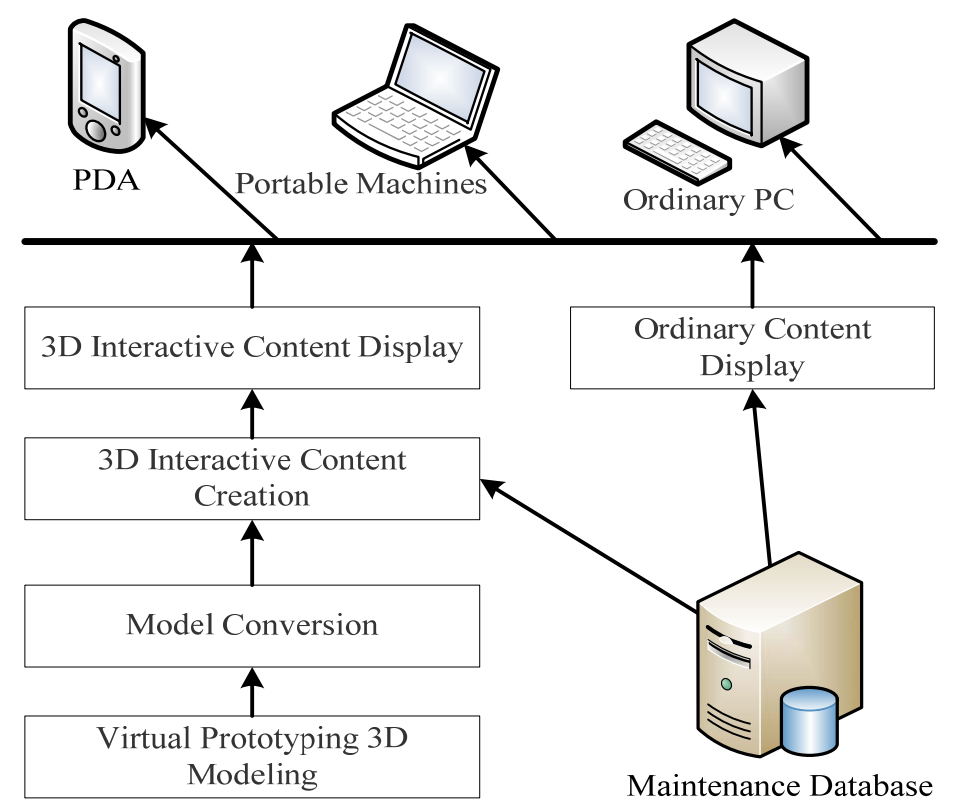

Fig.1 The framework of desktop virtual maintenance training platform

Virtual prototyping 3D modeling. The three-dimensional modeling of virtual maintenance is the basis of the method,the main work is how to build the virtual prototype 3D geometric modeling parts. The level of the assembly of parts should be restructed according to training mission requirements. Equipment - group - pieces - interact three-tier level structure object model can be established through simplifying virtual maintenance prototype model, ignoring the internal structures which are complex or not visible but no relationship with maintenance disassembly processes[8]. Common 3D modeling tools: 3ds Max, Maya, Pro / E, UG, SolidWorks, etc.

Model conversion module.Most of the geometrical models created by the three-dimensional modeling software are described by a continuous surface and volume composition, and are usually presented by mathematical methods. If these models are imported into content production module directly, which will make the complexity of the prototype is too high, we cannot meet the requirements of real-time rendering animate. Therefore, data format should be streamlined and converted before the content production module imported to 3D geometric model .The NGRAIN provide two models converter: Okino PolyTrans and TransMagic. Currently used 3D models can be converted to the NGRAIN specific .3 ko format using two converter [4].

3D interactive content creation module.3D interactive content production is mainly achieved by NGRAIN Producer authoring tools, mainly divided into two categories: one is to explain the principle construction equipment, dismantling and assembly process, common troubleshooting ,and some other training content, given in the form of animation, this mean can make trainees familiar structures and components attributes; another is operating practice , trainees select the instruments and tools, on the instruct of system boot and error to complete disassembly and assembly process, common troubleshooting training content, training people to enter the virtual maintenance free practice session.

\section{D interactive content display module.}

The main function of the 3D interactive content display module is to show good 3D interactive training content produced by 1.3 section, such as day-to-day maintenance and common troubleshooting including equipment operation method steps, routine maintenance process as well as machinery, electronic circuits, hydraulic oil common troubleshooting. 
Ordinary content display module.Ordinary content display module is mainly to show the structure and purpose of the equipment, the basic principles, such as functional description of the technical indicators, the main parameters of the equipment and a whole or a specific subsystem of the machine, electronic circuit, hydraulic oil works, etc.

Maintenance knowledge database.Collected mature case about the training of troops and equipment repair, and maintenance of knowledge, to provide the necessary material for content creation or display module.

\section{Desktop virtual maintenance training platform}

I realized dump truck desktop virtual maintenance training platform construction in the framework of the system shown in Figure 1,which can run on ordinary PC, portable tablet PCs and other client terminal.

The entire platform using the MySQL database storage dump truck ordinary content, 3D interactive content, and content generated in the system is running. The database can be called by users during virtual the process of maintenance training . The ordinary content and 3D interactive content integrated in DotNet environment. The integration of 3D interactive content is achieved by calling the standard COM control (Mobilizer) for primarily Windows environment provided by NGRAIN.

Shown in Fig.2, the trainees can complete equipment maintenance operations training content and master dump truck repair knowledge by inspecting the appearance picture of the equipment, the principle of different structures, and interactively 3D animation, operation training content. In this way, trainee's maintenance skills can be improved, and maintenance training cost-effective is greatly improved at last.

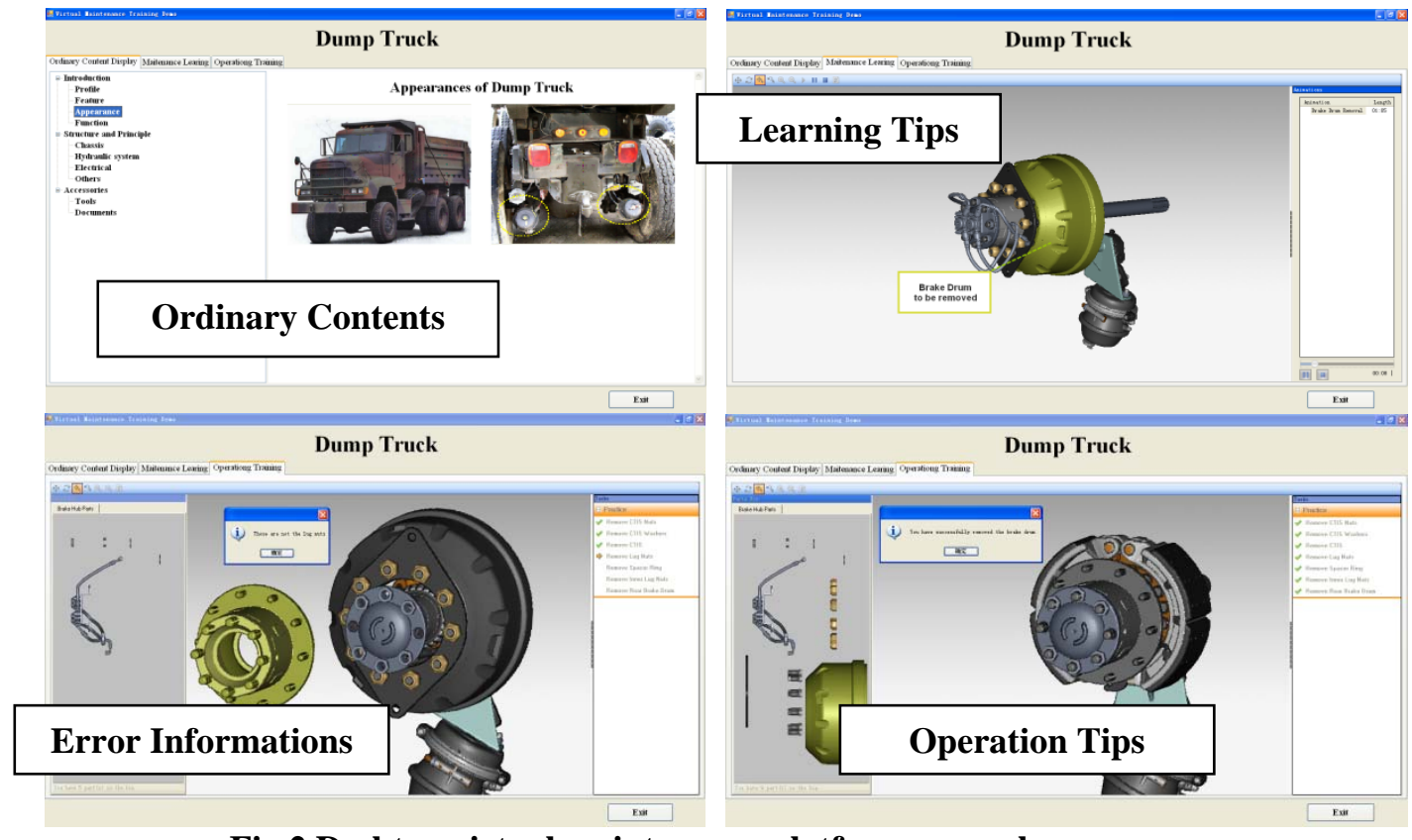

Fig.2 Desktop virtual maintenance platform examples

\section{Conclusion}

NGRAIN Producer is a interface friendly, publish, versatility desktop-level interactive applications software and suit for developers without programming or writing scripts, therefore common Microsoft Office users can quickly create and publish inter-active three-dimensional training courseware.

NGRAIN build desktop virtual service platform based on very low hardware requirements, the, the training mission will not be limited to a training room or a specific user, provides the possibility for the maintenance of the auxiliary system portability. It is a portable, economical solution for maintenance training. The system can not only meet the needs of the training mission, and also to 
provide a reference for the other virtual maintenance training system, and has a very important practical significance and broad application prospects.

In addition ,in the paper just take dump truck as a example, some exploratory work have been done, published interactive training material only, the management of the training process have not been realized in yet. In order to solve the above problem, the developer may be secondary development, complementary advantages, to achieve broader functional software module integration, improve the form or function of the maintenance training platform.

\section{References}

[1]Wang xiao-guang, Su Qun-xing. Key technology of immersion virtual maintenance and training system[J]. Ordnance Industry Automation, 2006(2): 33- 34.

[2] WANG Shang-jun, SHI He-ping, DOU Yun-jie. Design and Realization of Virtual Maintenance Training System for Certain Equipment[J]. Ordnance Industry Automation, 2009, 28(9): 11-14.

[3] LI J R, Khoo L P, T or S B. Desktop virtual reality for maintenance training: an object oriented prototype system ( v2 realism)[ J] . Computers in Industry, 2003, (52): 109- 125.

[4]LIANG Feng, ZHANG Zhi-li, LI Xiang-yang. Research on Development of Virtual Assembly Simulation Platform of Large-scale Armament Equipments[J]. Journal of System Simulation, 2009, 21(Suppl.2): 136-139.

[5] Kraus D, Gramopadhye A K. Team training: role of computers in the aircraft maintenance environment[J]. Computers \& Industrial Engineering, 1999, (36) : 635- 654.

[6]LIU Jin-lin, ZENG Fan-ming. Study on virtual maintenance training technology for the marine power plant[J]. SHIP SCIENCE AND TECHNOLOGY, 2008, 30(6):140-142.

[7] Liu Jia, Liu Yi. A Survey of Virtual Maintenance Technology[J]. JOURNAL OF COMPUTER-AIDED DESIGN\& COMPUTER GRAPHICS,2009, 21(11): 1519-1534.

[8] HAO Jin-ping, et.a1.Visual maintenance theory and technologies[M]. Beijing: National Defense Industry Press.2008:195-200.(In Chinese) 